\title{
Microalgae growth and oxygen production on different textile fabrics
}

\author{
Ewin Tanzli ${ }^{1}$, Bennet Brockhagen ${ }^{1}$, Aron Blümel', Inken Blanca Post ${ }^{2}$, Thorsten Bache ${ }^{2}$, \\ Khorolsuren Tuvshinbayar ${ }^{1}$, Sarah Vanessa Homburg ${ }^{1}$, Andrea Ehrmann ${ }^{1, *}$ \\ ${ }^{1}$ Bielefeld University of Applied Sciences, Faculty of Engineering and Mathematics, Bielefeld, Germany \\ 2 Bache GmbH, Rheinberg, Germany \\ ${ }^{*}$ Corresponding author E-mail address: andrea.ehrmann@fh-bielefeld.de
}

\section{INFO}

CDAPT, ISSN 2701-939X

Peer reviewed article

2022, Vol. 3, No. 1, pp. 9-16

DOI 10.25367/cdatp.2022.3.p9-16

Received: 23 November 2021

Accepted: 05 February 2022

Available online: 01 March 2022

\begin{abstract}
Microalgae can be used for diverse applications in research and industry. Several microalgae grow adhering to surfaces that are usually two-dimensional. A third dimension could increase the amount of microalgae adhering to a given area and can be offered by textile fabrics. Here we report on the microalgae Chlorella vulgaris and Scenedesmus spec. growing on different knitted fabrics under defined light and under office light conditions. Our results show a significant influence of illumination on both algal species and a smaller impact of the chosen medium, while all knitted fabrics under examination were found well suited as substrates. The numbers of alga cells per petri dish were higher on textile fabrics than in pure water or medium by a factor of $\sim 4-$ 20 , respectively.
\end{abstract}

\section{Keywords}

green microalgae,

knitted fabrics,

Tencel,

cotton,

linen,

oxygen production,

Clark electrode

(c) 2022 The authors. Published by CDAPT.

This is an open access article under the CC BY-NC-ND license https://creativecommons.org/licenses/ peer-review under responsibility of the scientific committee of the CDAPT.

(C) 2022 CDAPT. All rights reserved.

\section{Introduction}

Several green microalgae are highly interesting for research and industry. While some can be used for food, others are able to produce oxygen or hydrogen, can be used in pharmaceutics or for biofuel production etc. [1-5]. Microalgae are often grown in dispersion in photobioreactors of different shapes $[6,7]$; nevertheless, open ponds as well as closed bioreactors have the problem that the applied light intensity is only partly used, leading to high production costs. Thus, only high-value products from such algae are economically attractive. Strongly diluted suspensions that allow light from outside to enter the bioreactor, in contrast, cause problems in harvesting the algae. 
Growing algae on a substrate can overcome the harvesting problem [8]. Thus, one of the green microalgae most often investigated, Chlorella vulgaris, was examined in terms of adhesion properties on different rigid surfaces [9-11]. Generally, different polymers were found suitable for microalgae adhesion [12]. While using microcarriers did not improve harvesting [13], other groups concentrated on membranes and fibrous substrates. Using a woven fabric, C. vulgaris was successfully grown in a photobioreactor [14]. This solution was found to be better than a cotton rope and Spandex, as investigated in rotating drum and plate biofilm reactors [15].

In our group, previous studies showed good adhesion of another green microalga, Chlamydomonas reinhardtii, on electrospun nanofiber mats and other nonwovens [16]. For marine macroalgae [17] and $C$. vulgaris [18], growth on different knitted and woven fabrics was investigated, showing that especially jute woven fabrics were well suited for a high algae concentration. However, jute tends to get moldy and is thus not the perfect choice for a substrate for microalgae growth.

Here, we thus report on the growth of $C$. vulgaris and Scenedesmus spec. in flat bed reactors, measured by algae concentration on the fabrics as well as in the supernatant, and on their ability to produce oxygen when illuminated after cultivation for two weeks on different fabrics.

\section{Methods and materials}

The following flat-knitted fabrics were used to test the adhesion of green microalgae (Fig. 1):

- Plush fabric, 100\% Tencel, $2 \times \mathrm{Nm} 34 / 2,714 \mathrm{~g} / \mathrm{m}^{2}$, washed and ironed ("Plush Tencel")

- Plush fabric, $100 \%$ cotton, 4 x Nm 50/2, $918 \mathrm{~g} / \mathrm{m}^{2}$, washed and ironed ("Plush cotton")

- Plush fabric, $100 \%$ linen, 1 x Nm 6.6, $858 \mathrm{~g} / \mathrm{m}^{2}$, washed and ironed ("Plush linen")

- Relief jacquard structure, 100\% cotton, 2 x Nm 50/2 (“Jacquard”)
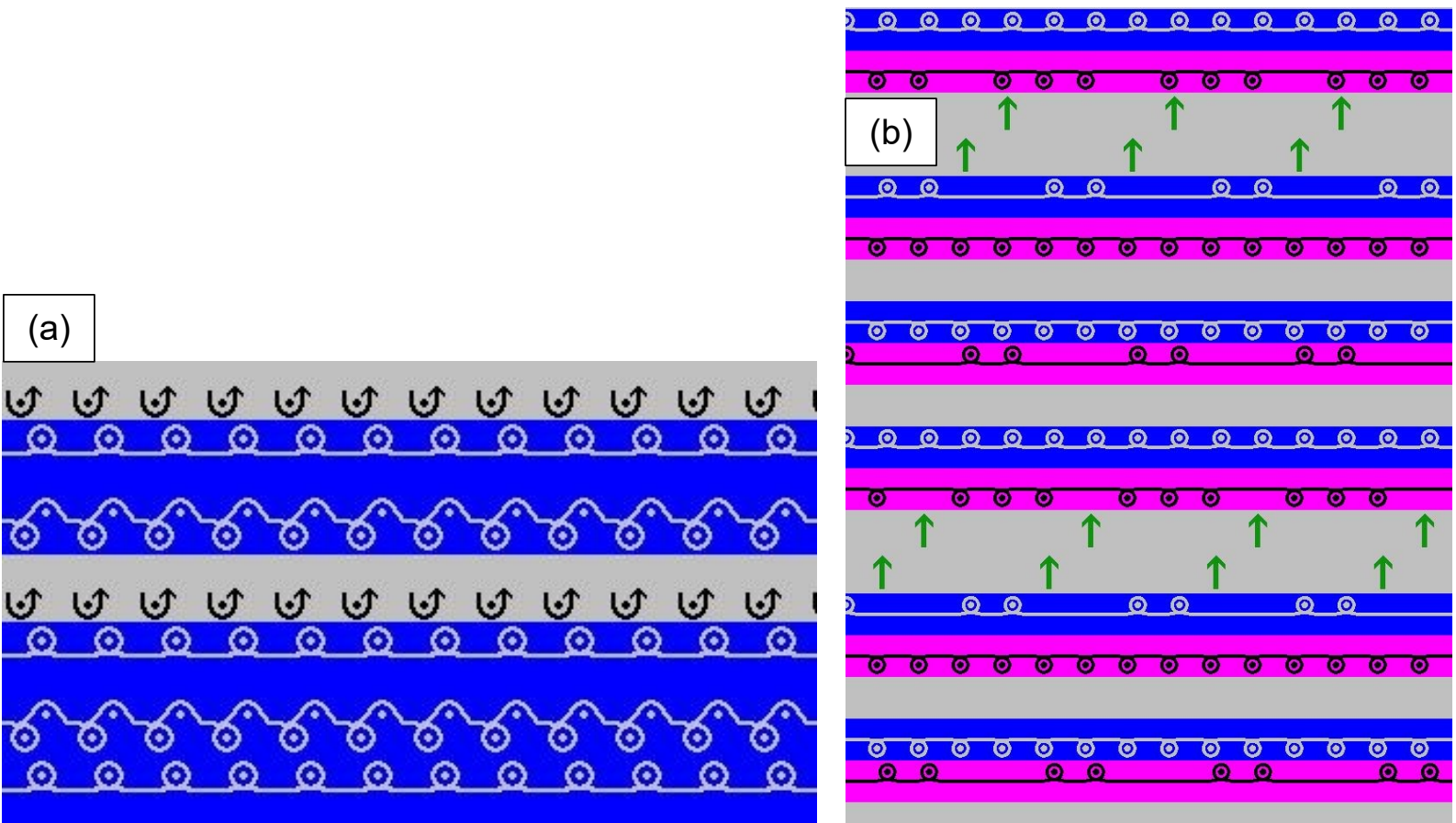

Fig. 1 Needle notations of (a) plush; (b) Jacquard fabric.

Microalgae C. vulgaris and Scenedesmus spec. (purchased from Planktino, Castrop-Rauxel, Germany) were cultivated on the textile fabrics placed in petri dishes (inner diameter $85 \mathrm{~mm}$ ) in triplicates. The medium contained phytoplankton fertilizer according to Guillard f/2 (Planktino, applied in a concentration of $1 \mathrm{~mL} / \mathrm{L}$ ). Colonization was started with $1 \mathrm{ml}$ parent solution containing $3.22 \cdot 10^{6}$ (C. vulgaris) and $1.24 \cdot 10^{8}$ (Scenedesmus) algae cells $/ \mathrm{ml}$, respectively, in $50 \mathrm{ml}$ medium. The petri dishes were partly illuminated by warm-white LED lights (CRI930 linear flex band, ISOLED, Schwoich, Austria) with an average intensity of $13-15 \mathrm{~W} / \mathrm{m}^{2}$ during a photoperiod of $12 \mathrm{~h} / \mathrm{d}$ ("illumination"). Other petri dishes were 
only illuminated by normal light available in an office in September/October 2021 in Germany, i.e. partly by artificial light ( 100 lux at the position of the petri dishes) and partly by sunlight ("no illumination"). The latter test was performed to investigate whether additional illumination of the algae is necessary or whether the available light is sufficient, which would help save energy during algae growth.

The algae were cultivated for 14 days; then the oxygen production rate was measured by a Clark electrode in an Oxygraph+ system (Hansatech Instruments, Norfolk, UK) for the supernatant, taken from the petri dishes, i.e. for the algae growing in suspension, and the parent solutions. The oxygen production per alga was calculated by the algal concentrations described below in the Clark electrode, identical to the concentrations in the supernatants and the parent solutions, respectively.

The algae adhering to the textile fabrics were completely separated from these fabrics using an ultrasonic device (UP200Ht, hielscher, Teltow, Germany). Algae suspensions of both species were counted undiluted and in dilutions of 1:1, 1:2, 1:3, 1:5 and 1:10 using an Improved Neubauer ruled hemocytometer (Paul Marienfeld $\mathrm{GmbH}$ \& Co. KG, Lauda Königshofen, Germany). These algae concentrations were also investigated by UV/Vis spectrometry (Genesys 10S, Thermo Fisher Scientific, Waltham, MA, USA) at a wavelength of $750 \mathrm{~nm}$, to calibrate UV/Vis absorbance with respect to algae concentration.

Microscopic images were taken by a fluorescence microscope Axio Observer in reflected light (Carl Zeiss Microscopy $\mathrm{GmbH}$, Oberkochen, Germany).

\section{Results and discussion}

Fig. 2 depicts microscopic images of $C$. vulgaris and Scenedesmus spec. cells in petri dishes and on textile fabrics. The images show the different dimensions of both algae (Figs. 2a, b) and their sizes in comparison to the fibers of the Tencel yarn used here.

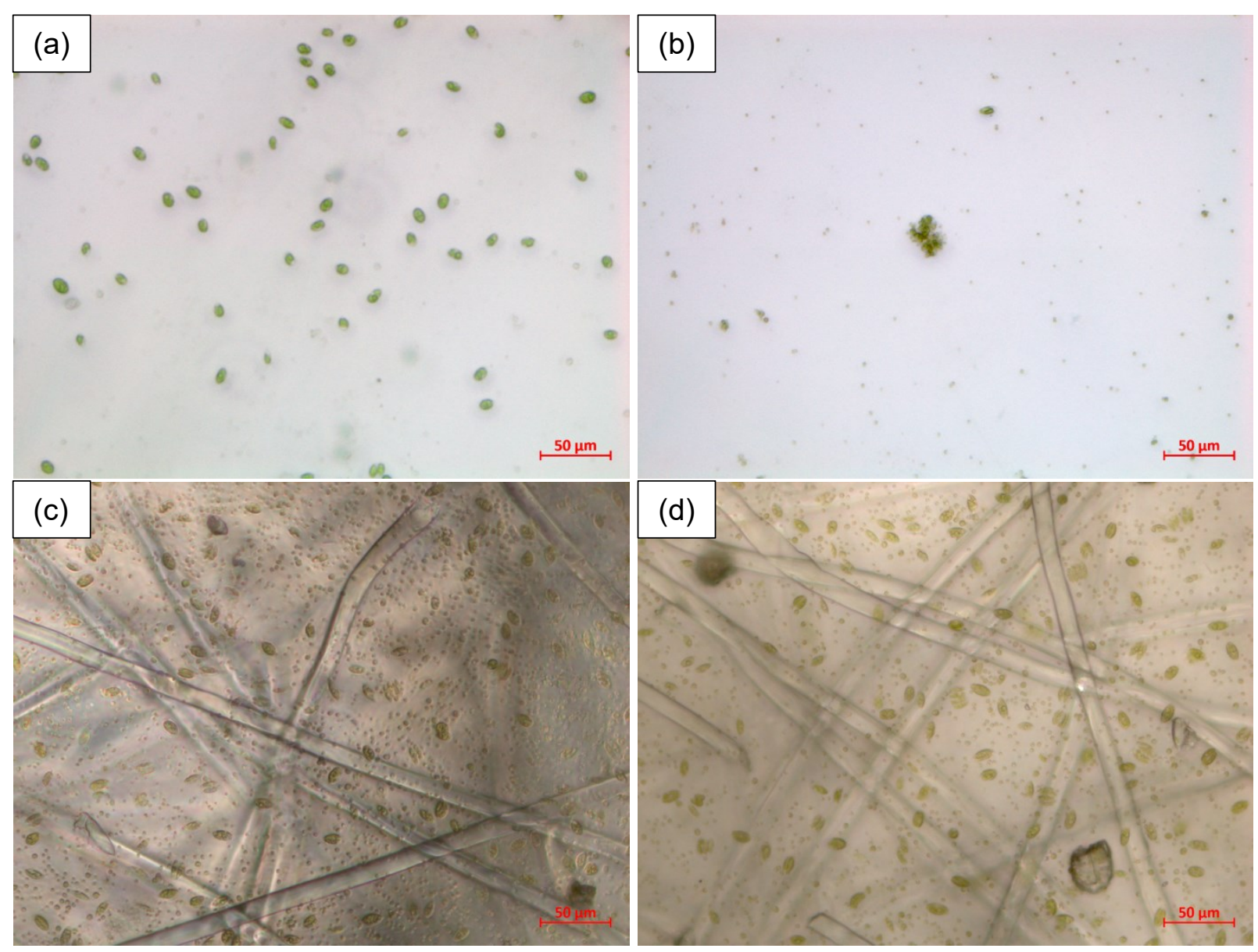

Fig. 2 Microscopic images of algal cells: (a) Scenedesmus spec. in a petri dish; (b) C. vulgaris in a petri dish; $(c, d)$ both species co-cultured on a plush Tencel fabric. 
Next, Fig. 3 gives a first overview of the impact of illumination and textile fabric on the growth of C. vulgaris in $\mathrm{f} / 2$ medium. While only a small and not significant influence of the textile material can be estimated, the influence of the illumination on the proliferation of algae cells is apparent. It should be mentioned that approx. one order of magnitude more cells are visible than at the beginning of cultivation (3.22 $10^{6}$ cells per petri dish). Besides, comparison with reference petri dishes (without textile fabric) shows a clear advantage of the large surface of the fabrics for adhering algae. This difference is even more pronounced for the situation without additional illumination.

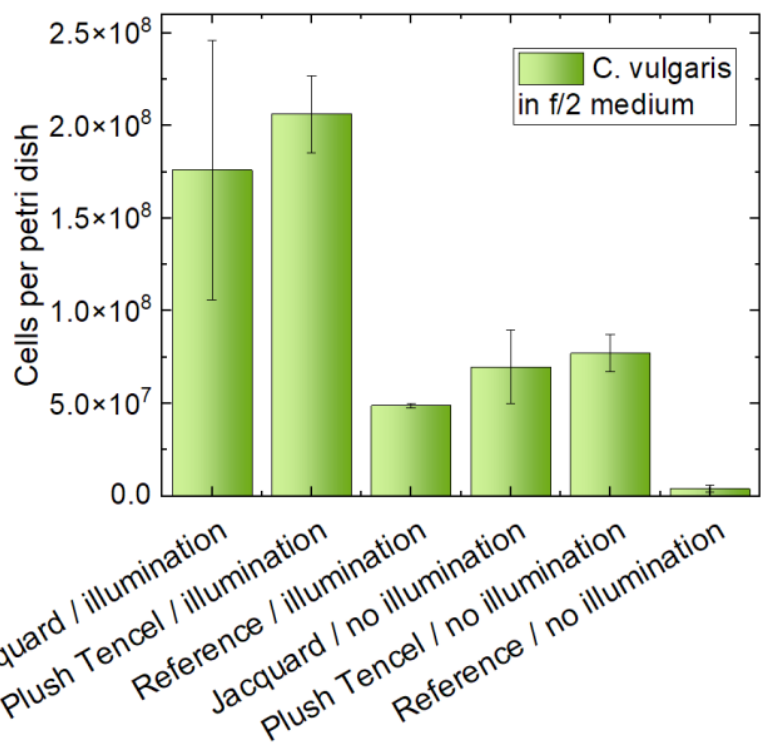

Fig. 3 Numbers of $\mathrm{C}$. vulgaris cells per petri dish after 14 days of cultivation in f/2 medium.

Next, Fig. 4 shows a comparison of the proliferation of C. vulgaris grown under illumination on different plush fabrics as well as in medium and water. Besides the cells on the textile fabrics, the cells found in the supernatant are also depicted here, indicating that indeed most algae grew on the textile fabrics, as it is necessary to use such fabrics for improved harvesting.

Comparing the fabrics knitted from different materials, only small differences are visible. Generally, the number of algae cells on plush linen is slightly smaller than that found on Tencel and cotton fabrics, while a comparison of Tencel and cotton does not show a clear advantage of one of these materials. Interestingly, the difference between cultivation on $\mathrm{f} / 2$ medium and water is not very large, either, thus in a next study, different amounts of $f / 2$ fertilizer should be investigated to find out which amount is necessary to ensure stable algae growth.
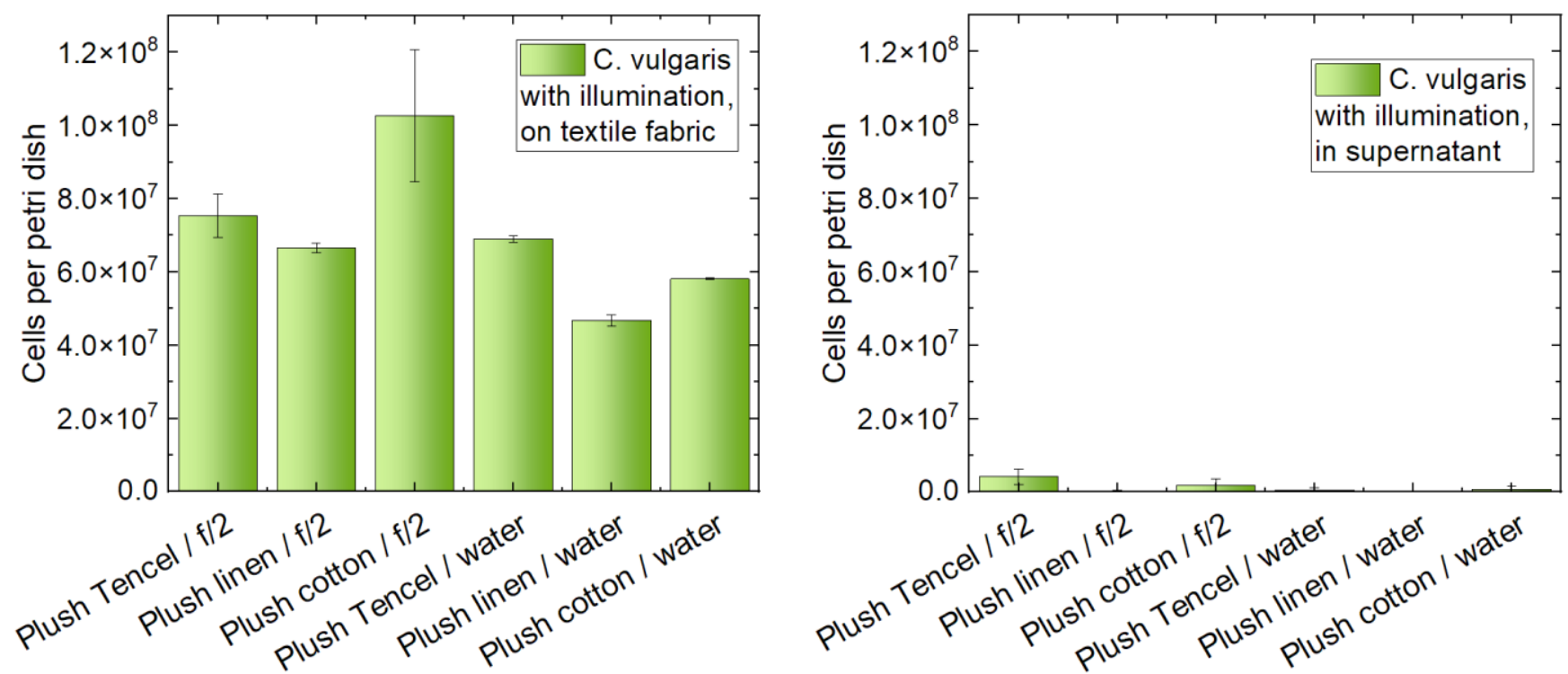

Fig. 4 Numbers of C. vulgaris cells per petri dish after 14 days of cultivation under illumination. 
In a next study, Scenedesmus spec. was investigated. Fig. 5 shows a comparison for $f / 2$ medium on the different plush fabrics with and without illumination. Generally, Scenedesmus spec. can be found in concentrations approx. 2 orders of magnitude larger per petri dish. This corresponds well with the approx. 2 orders of magnitude larger concentration of algae cells in the parent solution $\left(1.24 \cdot 10^{8}\right)$. The algae concentration thus increased by approx. one order of magnitude again here, such as for $C$. vulgaris.

Comparing Fig. 5 with Fig. 3, the effect of illumination is also similar, leading to an increase in algae concentration of approx. a factor 3 compared with the algae grown without illumination. Similar to Fig. 4, the amount of algae cells in the supernatant is again much smaller than on the knitted fabrics, while the numbers of algae in the references (without textile fabrics) are significantly smaller than in the petri dishes with textile fabrics.
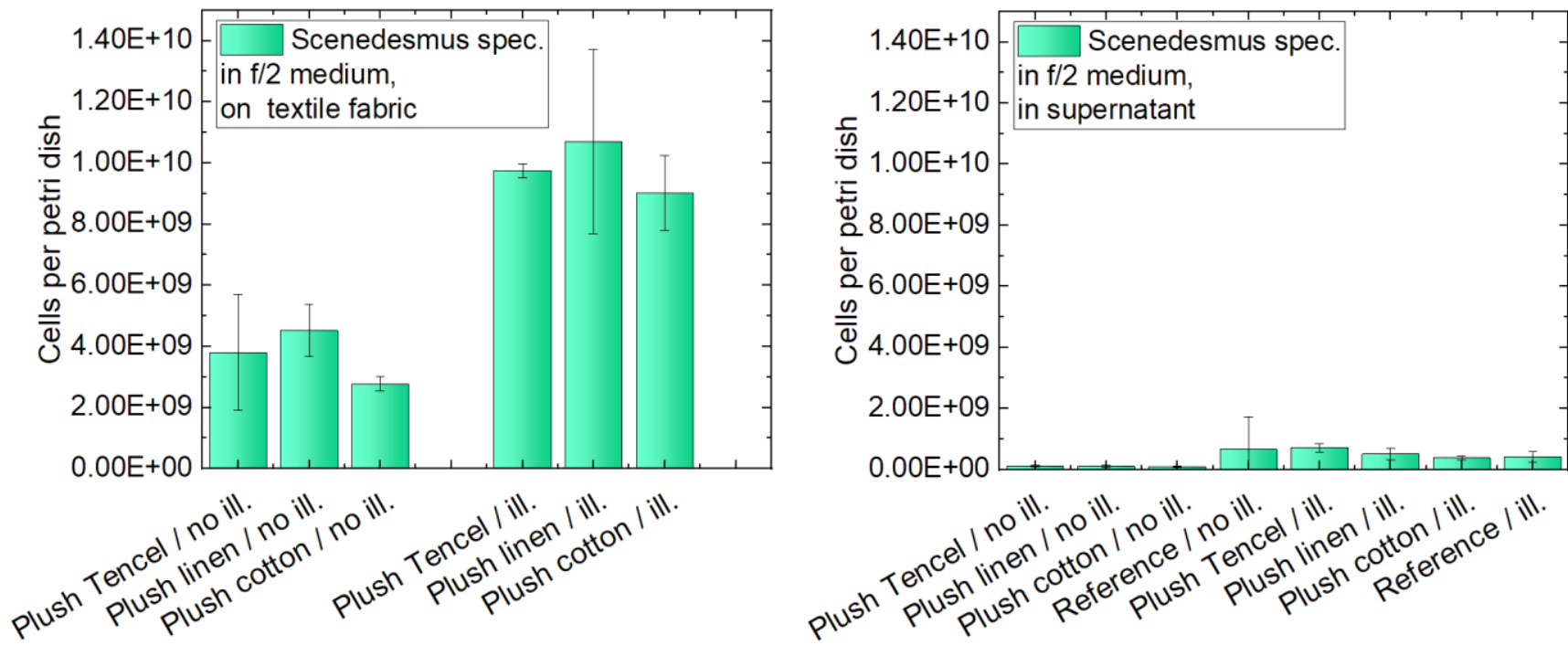

Fig. 5 Numbers of Scenedesmus spec. cells per petri dish after 14 days of cultivation under illumination.

It must be mentioned that the microalgae adhere to the textile fabrics under investigation very well. Fig. 6 shows examples of Tencel fabrics with $C$. vulgaris and Scenedesmus spec. gently washed under running water for 10 minutes, showing that even after this time, residual algae can still be found on the textile fabrics. Videos of the algae being washed from Tencel plush with more pressure can be found in the supplementary information.
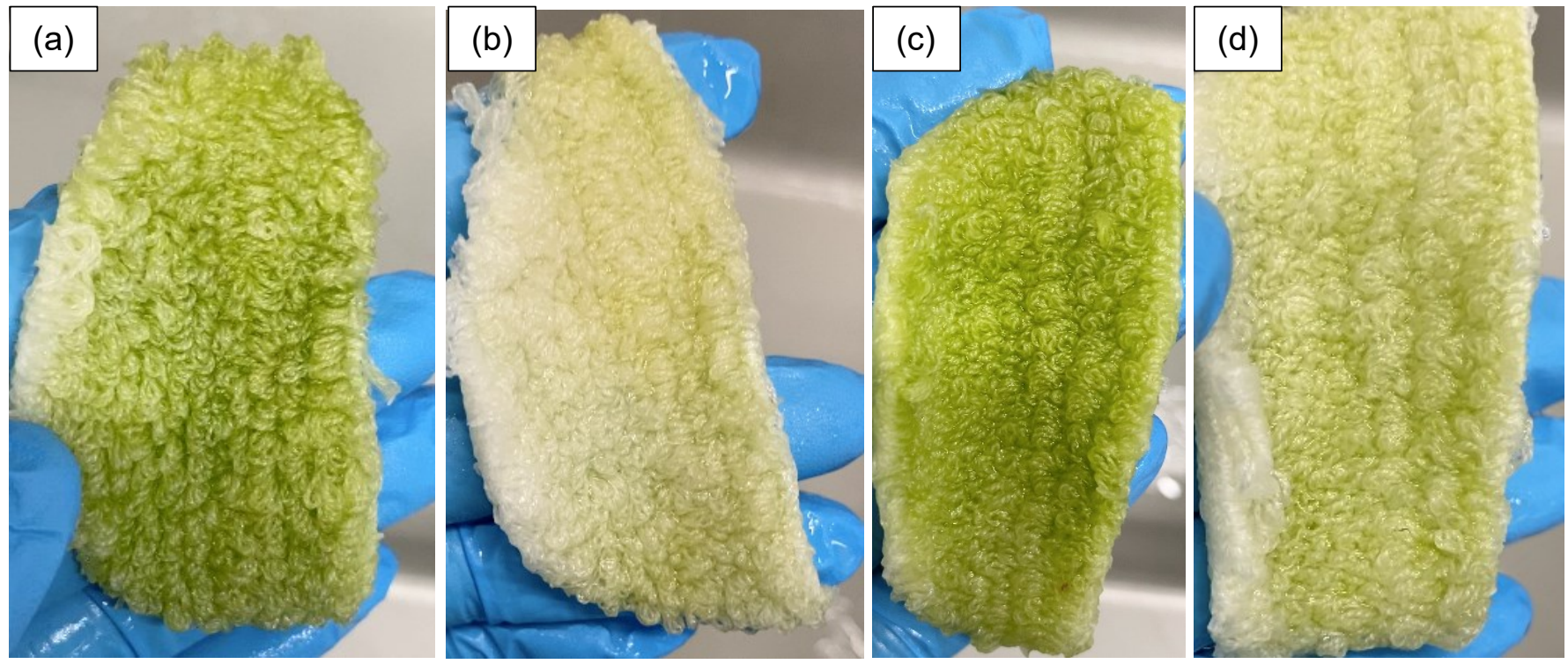

Fig. 6 (a) C. vulgaris on a Tencel plush; (b) after 10 min of gentle washing; (c) Scenedesmus spec. on a Tencel plush; (d) after 10 min of gentle washing. 
While the aforementioned parameters - material and structure of the textile fabric, medium and illumination - apparently have a very similar influence on both green algae, there seems to be one major difference between both algal species, as depicted in Fig. 7. Only the Scenedesmus spec. produces a discernible amount of oxygen, shown here for a petri dish without textile fabric for better visibility. In contrast, comparison of the algal concentrations shows that the starting concentration of Scenedesmus spec. is already one order of magnitude higher than the final concentration of $C$. vulgaris, indicating that the lower amount of visible bubbles on $C$. vulgaris cultures may be based on their low concentration.

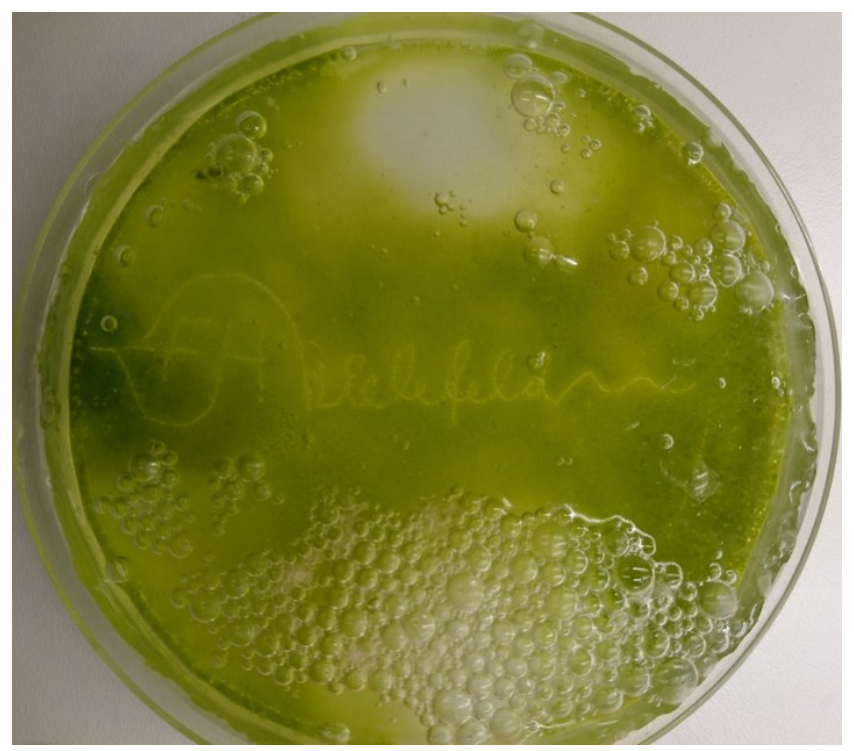

Fig. 7 Oxygen bubbles produced by Scenedesmus spec. after 14 days of cultivation.

To verify whether Scenedesmus spec. produces more oxygen than $C$. vulgaris, the oxygen production rate was measured using a Clark electrode. For this, only the algae from the supernatants were used since separating the algae from the textile fabrics by an ultrasonic device destroyed them and made subsequent oxygen measurements impossible. Fig. 8 shows exemplary results, comparing the parent solutions of both algae under examination as well as the references. In the latter, the algae seem to be more active, producing more oxygen. In contrast to the visual impression of the petri dishes (cf. Fig. 6), the objective measurement shows that $C$. vulgaris produces more oxygen per algal cell than Scenedesmus spec. This result suggests letting especially C. vulgaris grow in denser cultures, as enabled by the textile substrates, to produce more oxygen per culture volume.

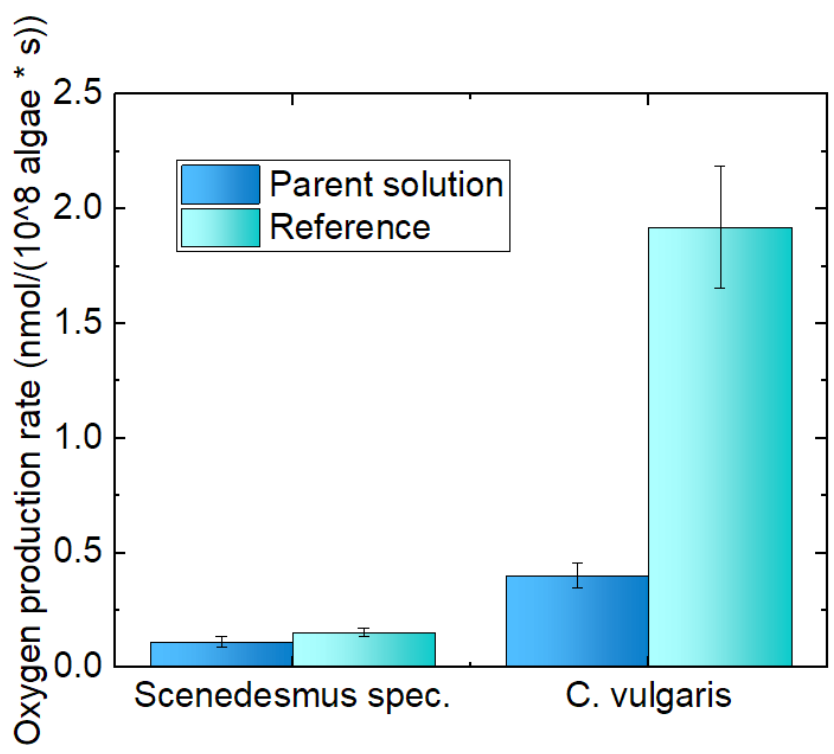

Fig. 8 Oxygen production rates for Scenedesmus spec. and C. vulgaris parent solutions and reference samples, i.e. petri dishes without textile fabrics. 


\section{Conclusions and outlook}

In a recent study, the green microalgae $C$. vulgaris and Scenedesmus spec. were cultured on different knitted fabrics. The results show a strong impact of illumination as well as a certain influence of the chosen medium on both algal species. The knitted fabrics used in this study, prepared from different natural fibers, all showed good algal cell adhesion and proliferation, with a significantly higher number of algae growing on the fabrics than found in the supernatant.

Future studies will aim at finding optimum concentrations to produce a maximum amount of oxygen from high-density cultures under different light conditions.

\section{Author Contributions}

E. Tanzli: conceptualization, investigation, writing - review and editing; Bennet Brockhagen: conceptualization, investigation, writing - review and editing; Aron Blümel: investigation, writing - review and editing; Inken Blanca Post: investigation, writing - review and editing; Thorsten Bache: investigation, writing - review and editing; Khorolsuren Tuvshinbayar: investigation, writing - review and editing; Sarah Vanessa Homburg: conceptualization, methodology, writing - review and editing; Andrea Ehrmann: conceptualization, writing - original draft preparation, visualization. All authors have read and agreed to the published version of the manuscript.

\section{Acknowledgements}

This project was funded by the Federal Ministry for Economic Affairs and Energy in the scope of the ZIM project KK5129704CS0. The authors acknowledge funding of the fluorescence microscope by the Ministry of Culture and Science of the State of North Rhine-Westphalia by the Zukunftsfonds. We would like to thank Ana-Katrina Büttner for language editing.

\section{Conflicts of Interest}

The authors declare no conflict of interest.

\section{References}

[1] González-Fernández, C.; Sialve, B; Bernet, N.; Steyer, J. P. Impact of microalgae characteristics on their conversion to biofuel. Part I: focus on cultivation and biofuel production. Biofuel Bioprod. Biorefin. 2012, 6, 105-113. DOI 10.1002/bbb.338.

[2] Homburg, S. V.; Kruse, O.; Patel, A. V. Growth and photosynthetic activity of Chlamydomonas reinhardtii entrapped in lens-shaped silica hydrogels. J. Biotechnol. 2019, 302, 58-66. DOI 10.1016/j.jbiotec.2019.06.009.

[3] Homburg, S. V.; Venkanna, D.; Kraushaar, K.; Kruse, O.; Kroke, E.; Patel, A. V. Entrapment and growth of Chlamydomonas reinhardtii in biocompatible silica hydrogels. Coll. Surf. B Biointerfaces 2019, 173, 233-241. DOI 10.1016/j.colsurfb.2018.09.075.

[4] Fradique, M.; Batista, A. P.; Nunes, M. C.; Gouveia, L.; Bandarra, N. M.; Raymundo, A. Incorporation of Chlorella vulgaris and Spirulina maxima biomass in pasta products. Part 1: preparation and evaluation. J. Sci. Food Agric. 2010, 90, 1656-1664. DOI 10.1002/jsfa.3999.

[5] Becker, W. Microalgae in human and animal nutrition. In Handbook of microalgal culture; Richmond, A., Ed.; Blackwell, Oxford, 2004; pp 312-351.

[6] Borowitzka, M. A. Commercial production of microalgae: ponds, tanks, tubes and fermenters. J. Biotechnol. 1999, 70, 313-321. DOI 10.1016/S0168-1656(99)00083-8.

[7] Lee, Y. K. Microalgal mass culture systems and methods: Their limitation and potential. J. Appl. Phycol. 2001, 13, 307-315. DOI 10.1023/A:1017560006941.

[8] Ozkan, A.; Kinney, K.; Katz, L.; Berberoglu, H. Reduction of water and energy requirement of algae cultivation using an algae biofilm photobioreactor. Bioresour. Technol. 2012, 114, 542-548. DOI 10.1016/j.biortech.2012.03.055.

[9] Sekar, R.; Venugopalan, V.; Satpathy, K.; Nair, K. V. K.; Rao, V. N. R. Laboratory studies on adhesion of microalgae to hard substrates. Hydrobiologia 2004, 512, 109-116. DOI 10.1023/B:HYDR.0000020315.40349.38.

[10] Johnson, M. B.; Wen, Z. Y. Development of an attached microalgal growth system for biofuel production. Appl. Microbiol. Biotechnol. 2010, 85, 525-534. DOI 10.1007/s00253-009-2133-2. 
[11] Ozkan, A.; Berberoglu, H. Adhesion of algal cells to surfaces. Biofouling 2013, 29, 469-482. DOI 10.1080/08927014.2013.782397.

[12] Melo, M.; Fernandes, S.; Caetano, N.; Borges, M. T. Chlorella vulgaris (SAG 211-12) biofilm formation capacity and proposal of a rotating flat plate photobioreactor for more sustainable biomass production. J. Appl. Phycol. 2018, 30, 887-899. DOI 10.1007/s10811-017-1290-4

[13] Gao, F.; Yang, Z.-H.; Li, C; Zeg, G.-M.; Ma, D.-H.; Zhou, L. A novel algal biofilm membrane photobioreactor for attached microalgae growth and nutrients removal from secondary effluent. Bioresour. Technol. 2015, 179, 8-12. DOI 10.1016/j.biortech.2014.11.108.

[14] Shen, Y.; Yang, T.; Zhu, W.; Zhao, Y. Wastewater treatment and biofuel production through attached culture of Chlorella vulgaris in a porous substratum biofilm reactor. J. Appl. Phycol. 2017, 29, 833-841. DOI 10.1007/s10811-016-0981-6.

[15] Shen, Y.; Zhu, W.; Chen, C.; Nie, Y.; Lin, X. Biofilm formation in attached microalgal reactors. Bioproc. Biosyst. Eng. 2016, 39, 1281-1288. DOI 10.1007/s00449-016-1606-9.

[16] Großerhode, C.; Wehlage, D.; Grothe, T.; Grimmelsmann, N.; Fuchs, S.; Hartmann, J.; Mazur P.; Reschke, V.; Siemens, H.; Rattenholl, A.; Homburg, S. V.; Ehrmann, A.) Investigation of microalgae growth on electrospun nanofiber mats. AIMS Bioeng. 2017, 4, 376-385. DOI 10.3934/bioeng.2017.3.376.

[17] Sebök, S.; Brockhagen, B.; Storck, J. L.; Post, I. B.; Bache, T.; Korchev, R.; Böttjer, R.; Grothe, T.; Ehrmann, A. Growth of marine macroalgae Ectocarpus sp. on various textile substrates. Environmental Technology 2020, online first. DOI 10.1080/09593330.2020.1829086.

[18] Brockhagen, B.; Storck, J. L.; Grothe, T.; Böttjer, R.; Ehrmann, A. Improved growth and harvesting of microalgae Chlorella vulgaris on textile fabrics as 2.5D substrates. AIMS Bioeng. 2020, 8, 16-24. DOI 10.3934/bioeng.2021003. 